\title{
INVESTIGATING THE RELEVANCE OF DISSOCIATION TO PSYCHOSIS
}

Aggelopoulou E, Tzikos A, Tsaltabasidou E, Nehnentidou F, Kafalis G Department of Psychiatry University General Hospital of Alexandroupolis Alexandroupolis Greece

\section{OBJECTIVES}

Increasing evidence suggests the connection between dissociation and psychosis. Significant rates of dissociative symptoms are detected in psychotic disorders and chronic schizophrenia. So far, no study has adequately investigated if these symptoms preexisted the first episode. This review aims to examine the possibility of preventing psychotic episodes through dealing with dissociative symptoms and including them into treatment planning.

\section{BACKGROUND AND AIMS}

We conducted an extended review of the literature investigating the prevalence of dissociative symptoms in psychotic episodes. We initially looked at the possible overlapping of dissociative and psychotic symptoms. Then we examined whether a predisposition to psychosis could exist in dissociative disorder patients. Could an early intervention plan be developed in order to lower the risk of a psychotic episode later on?

\section{METHOD}

Several databases, including PubMed and Scopuxs were searched for apposite literature. We found articles that dealt with our project through collecting interviews, questionnaires and information about experiences of dissociation, focusing on first-episode psychosis (FEP) patients.

RESULTS

Preliminary findings show that high ratio of psychotic patients have experienced dissociative symptoms, specifically depersonalization. The development of positive symptoms (i.e. hallucinations and delusions) and the disruption in the function of consciousness, memory, identity and perception characterizing dissociation may share a potentially similar mechanism which remains unclear. Recent theoretical proposals added to this, assuming there is a proneness to hallucinations among people with dissociative experiences.

\section{CONCLUSIONS}

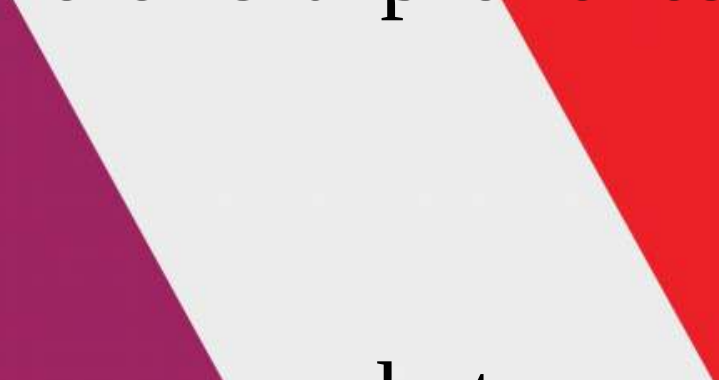

High levels of co-occurence between psychotic disorders and dissociation were indicated. Dissociative symptoms should be detected and evaluated. An early intervention could be a useful addition to a traditional treatment plan. Further research is required to explore a possible improvement on prognosis. 